\title{
Association between Self-Reported Exposure to Alcohol Advertisements and Drinking Behaviors: An Analysis of a Population-Based Survey in Thailand
}

\author{
Phagapun Boontem ${ }^{1}$ and Udomsak Saengow $2,3,4, *$ (D) \\ 1 Faculty of Nursing, Srinakharinwirot University, Nakhon Nayok 26120, Thailand; phagapun@g.swu.ac.th \\ 2 Research Institute for Health Sciences, Walailak University, Nakhon Si Thammarat 80160, Thailand \\ 3 Center of Excellence in Data Science for Health Study, Walailak University, \\ Nakhon Si Thammarat 80160, Thailand \\ 4 School of Medicine, Walailak University, Nakhon Si Thammarat 80160, Thailand \\ * Correspondence: udomsak.sa@wu.ac.th
}

Citation: Boontem, P.; Saengow, U. Association between Self-Reported Exposure to Alcohol Advertisements and Drinking Behaviors: An Analysis of a Population-Based Survey in Thailand. Int. J. Environ. Res. Public Health 2021, 18, 11271. https:/ / doi.org/10.3390/ijerph182111271

Academic Editor: Paul B. Tchounwou

Received: 9 September 2021

Accepted: 25 October 2021

Published: 27 October 2021

Publisher's Note: MDPI stays neutral with regard to jurisdictional claims in published maps and institutional affiliations.

Copyright: (C) 2021 by the authors Licensee MDPI, Basel, Switzerland. This article is an open access article distributed under the terms and conditions of the Creative Commons Attribution (CC BY) license (https:/ / creativecommons.org/licenses/by/ $4.0 /)$.

\begin{abstract}
The relationship between alcohol advertising and drinking has been demonstrated in many studies. Most studies were conducted on adolescents or young adults. Thailand has strict regulations on alcohol advertisements. This study aimed to examine associations between exposure to alcohol advertisements and drinking behaviors, i.e., past-year drinking and past-year heavy drinking, using data from a population-based survey in Thailand. The survey participants were Thai citizens aged 15 or older. Logistic regression was used to investigate the associations. The primary explanatory variable was self-reported exposure to alcohol advertisements. Covariates in the regression models included sex, age, and education. Self-reported exposure to alcohol advertisements was associated with past-year drinking (OR, 1.16; 95\% CI, 1.07-1.27), past-year heavy drinking (OR, 1.35; 95\% CI, 1.28-1.41), and past-year heavy drinking among drinkers (OR, 1.51; 95\% CI, 1.43-1.60). Male sex, working age, and secondary education or a diploma were associated with higher odds of past-year drinking and past-year heavy drinking. In this study, self-reported exposure to alcohol advertisements was shown to be associated with past-year drinking and past-year heavy drinking among a population aged 15 years or older.
\end{abstract}

Keywords: alcohol advertisement; alcohol advertising exposure; alcohol consumption; heavy drinking; association; cross-sectional study

\section{Introduction}

Alcohol consumption is attributed to more than 200 diseases and injuries. Health consequences related to alcohol use include maternal health, child development, cancer, cardiovascular diseases, injuries, violence, mental health, TB, and HIV/AIDS, among others. In 2016, there were 3 million alcohol-related deaths worldwide. In the same year, alcohol was responsible for 132.6 million disability-adjusted life years (DALYs). Premature death accounted for more than $80 \%$ of the DALYs associated with alcohol [1].

Studies from several countries found a positive association between exposure to alcohol advertisements and alcohol consumption. A survey of 909 adolescents from Chile reported a $41 \%$ increase in the likelihood of being drinkers and an $85 \%$ increase in the risk of being problematic drinkers in adolescents with recent exposure to alcohol advertisements [2]. The daily or almost daily exposure to alcohol advertisements was associated with current alcohol use (odds ratio (OR), 1.6; 95\% confidence interval (CI), 1.0-2.5) and drunkenness (OR, 2.3; 95\% CI, 1.4-3.8) according to a cross-sectional study of 3806 school children from Cambodia [3]. A household survey of 955 South Africans aged 18-65 years old found that alcohol advertising using SMS and free alcohol offers was associated with heavy drinking [4]. A cross-national survey of 9038 school children 
from Germany, Italy, the Netherlands, and Poland demonstrated that exposure to online alcohol advertising and owning alcohol-branded items increased the likelihood of ever use of alcohol and past-month binge drinking [5]. An online survey of 3399 UK adolescents found that a higher level of past-month exposure to alcohol marketing was associated with a higher-risk drinking pattern. Owning alcohol-branded items was associated with a higher-risk drinking pattern among drinkers and a higher susceptibility to drink among never drinkers [6]. A recent large study of 54,671 US adults aged 21 years and older found that a higher volume of alcohol advertising exposure was associated with past-month consumption of alcohol and, among past-month drinkers, more drinks consumed [7]. In addition to the evidence from these cross-sectional studies, a systematic review of 12 cohort studies regarding alcohol consumption in youths found that the level of alcohol marketing exposure was positively associated with alcohol use and a higher-risk drinking pattern among youths [8]. Regarding the rise in digital marketing, a recent systematic review found that engagement in digital alcohol marketing activities was associated with alcohol consumption [9]. Hence, a relationship between alcohol advertising and drinking was demonstrated in studies from various countries. The majority of studies focused on adolescents or young adults.

In Thailand, regulation of alcohol advertising and marketing is included as a measure under the altering social norms toward alcohol and reducing drinking motivation strategy, one of the five strategies of the 2010 National Alcohol Strategy [10]. The primary law regulating alcohol advertising and marketing is the Alcoholic Beverage Control Act, B.E. 2551 (2008). Following the enactment of the Alcoholic Beverage Control Act, B.E. 2551 (2008), most types of alcohol advertising in Thailand have been banned. Section 32 of the act [11], which is a regulation of alcohol advertising, states

"No person shall advertise or display, directly or indirectly, the name or trademark of any alcoholic beverage in a manner showing the properties thereof or inducing another person to drink.

Advertisements or public relations provided by the manufacturer of any kind of alcoholic beverage shall only be made for giving information thereof or giving social creative knowledge without displaying any illustration of such alcoholic beverage or its package, except for the display of a symbol of such alcoholic beverage or that of its manufacturer as prescribed by the Ministerial Regulation.

The provisions of paragraph one and paragraph two shall not apply to any advertisement broadcast from outside of the Kingdom."

The act effectively prohibits all direct advertising of alcoholic beverages. It only allows for a brief presentation of the logo with messages unrelated to drinking. Recently, surrogate advertising has been used by the alcohol industry in Thailand. Soft drink logos (such as drinking water, soda, and flavored soft drinks) were recently changed to resemble the logos of alcoholic beverages owned by the same conglomerate [12]. These soft drinks are promoted in place of alcoholic beverages through all media channels, including television, newspapers, billboards, and social media. In addition to the regulation, there is an annual social movement campaign encouraging three months of sobriety, in which millions of drinkers participate [13]. This campaign aims to counteract the effect of advertising and marketing on altering norms toward drinking. It is included in the 2010 National Alcohol Strategy under the same strategy as the regulation of alcohol advertising and marketing [10].

In the context of the Thai alcohol policy regarding alcohol advertising described above, this study aimed to examine the association between exposure to alcohol advertisements and drinking behaviors (i.e., past-year drinking and past-year heavy drinking) using data from a population-based survey of Thai citizens aged 15 years or older. 


\section{Materials and Methods}

\subsection{Study Design}

This study was an analysis of data from the 2017 Smoking and Drinking Behavior Survey (SADBeS17), which was conducted by the National Statistical Office, Thailand. Data from part III of the survey, which was related to alcohol consumption and its consequences, were used in the analysis. The protocol of this study was approved by the Human Research Ethics Committee of Walailak University, Nakhon Si Thammarat, Thailand (WU-EC-MD-3-473-63).

\subsection{Data Source}

The SADBeS17 is a population-based survey of Thai citizens aged 15 or older. The stratified two-stage sampling technique was used. Each province was considered a stratum. There were a total of 77 strata. In each stratum, 2315 enumeration areas were chosen at random from a total of 129,440 enumeration areas. Households were randomly selected from the enumeration areas.

The eligibility criteria included being 15 years old or older, having Thai citizenship, and being able to communicate fluently in Thai. All eligible individuals in selected households were invited to participate in the survey. Informed consent was obtained from all survey participants. For participants aged less than 18 years, consent was given by their guardians and themselves. The total number of survey participants was 92,015 . The response rate was $93.5 \%$. There were 89,154 participants with complete data. The analysis included only records with complete data. The data were collected between May and July 2017.

The survey items included demographic characteristics (age, sex, nationality, education level, marital status, occupational, etc.), smoking-related items, and alcohol-related items. The outcome variables in this study were drinking and past-year heavy drinking. Self-reported exposure to alcohol advertisements was the main explanatory variable. Covariates included sex, age, and education level. These covariates were selected as they are recognized determinants of alcohol consumption [1].

\subsection{Data Management}

The variables used in this analysis included drinking status, past-year heavy drinking, self-reported exposure to alcohol advertisements, sex, age group, and education level.

The primary outcome was past-year drinking. The item for past-year drinking was "Had you ever drunk alcoholic beverages in the past 12 months?" The responses were classified into three levels: no, occasionally (once a month or less frequently), and regularly (at least once a week). Participants who drank occasionally or regularly were classified as past-year drinkers based on their responses to this item. The secondary outcome was pastyear heavy drinking. The item for past-year heavy drinking was "How often had you drunk heavily ( 5 drinks or more) in a short period of time in the past 12 months?" The responses were classified into three levels: no, occasionally (once a month or less frequently), and regularly (at least once a week). Both drinking variables were dichotomized in order to be used as dependent variables in the binary logistic regression. The responses "occasionally" and "regularly" were categorized as "yes", while the response "no" remained unchanged.

Self-reported exposure to alcohol advertisements was the main explanatory variable. The item was "Had you been exposed to alcohol advertisements in the last 30 days?" There were three possible responses to this question: yes, no, and uncertain. There was no further explanation provided regarding alcohol advertising. Hence, participants considered what they perceived to be alcohol advertisements by themselves. The sex variable had two levels: male and female. Age was classified into five categories: 15-19, 20-30, 31-45, 46-60, and $61+$. There were four levels of education: primary education or lower level, secondary education or diploma, bachelor's degree or higher, and missing. 


\subsection{Statistical Analysis}

Survey participants were described using descriptive statistics (mean, standard deviation, and percentage). The percentages of past-year drinkers, past-year heavy drinkers (among the total population), and past-year heavy drinkers among drinkers were computed. Binary logistic regression was performed to examine the relationship between self-reported exposure to alcohol advertisements and both drinking variables (i.e., past-year drinking and past-year heavy drinking).

The regression model for past-year drinking had self-reported exposure to alcohol advertisements, sex, age, and education level as covariates (Model 1). Data from 89,154 participants with complete information were used in this model. For past-year heavy drinking, two regression models were performed (Model 2 and Model 3). Both models had the same set of covariates, including self-reported exposure to alcohol advertisements, sex, age, and education level. Model 2 examined the association between self-reported exposure to alcohol advertisements and past-year heavy drinking among the total participants. As in Model 1, data from all participants with complete information were used. Model 3 examined an association between self-reported exposure to alcohol advertisements and past-year heavy drinking among past-year drinkers. Data from 23,073 past-year drinkers were used in Model 3. Odds ratios (ORs) and 95\% CIs were estimated from the regression models to indicate the magnitude of associations. The level of significance was set at $5 \%$. All analyses were unweighted. The $R$ statistical language version 4.0 .3 was used to perform the statistical analysis.

\section{Results}

\subsection{Characteristics of Participants}

Over half of the participants were women. The average age was 47.5 years old. More than half attained primary education or lower, whereas $11.3 \%$ attained a bachelor's degree or higher. One third had been exposed to alcohol advertisements; $3.8 \%$ could not recall whether they had been exposed or not. One fourth were past-year drinkers; $11.2 \%$ drank regularly in the past year. About $10 \%$ of the participants were past-year heavy drinkers; more than half of the heavy drinkers regularly engaged in heavy drinking episodes (Table 1).

Table 1. Characteristics of participants in the analysis.

\begin{tabular}{lrr}
\hline \multicolumn{1}{c}{ Characteristic } & Frequency & \% \\
\hline Sex & & \\
Male & 40,724 & 45.7 \\
Female & 48,430 & 54.3 \\
Age (years) & \multicolumn{1}{c}{ Mean $=47.5(\mathrm{SD}=17.5)$} \\
$15-19$ & 11,458 & 6.8 \\
$20-30$ & 22,510 & 12.9 \\
$31-45$ & 27,757 & 25.2 \\
$46-60$ & 21,383 & 31.1 \\
61+ & & 24.0 \\
Education level & 49,500 & \\
Primary education or lower & 29,336 & 55.5 \\
Secondary education or diploma & 10,093 & 32.9 \\
Bachelor's degree or higher & 225 & 11.3 \\
Missing & & 0.3 \\
Self-reported exposure to alcohol advertisements & 29,128 & \\
Exposed & 56,637 & 32.7 \\
Not exposed & 3389 & 63.5 \\
Uncertain & & 3.8 \\
\hline
\end{tabular}


Table 1. Cont.

\begin{tabular}{lcc}
\hline \multicolumn{1}{c}{ Characteristic } & Frequency & $\%$ \\
\hline Past-year drinking & & \\
No & 66,081 & 74.2 \\
Occasionally & 13,057 & 14.6 \\
Regularly & 10,016 & 11.2 \\
Past-year heavy drinking & & \\
No & 79,927 & 89.65 \\
Occasionally & 3943 & 4.42 \\
Regularly & 5284 & 5.93 \\
\hline
\end{tabular}

SD, standard deviation.

\subsection{Past-Year Drinkers and Past-Year Heavy Drinkers}

The proportion of past-year drinkers in men was considerably higher than in women at $45.1 \%$ versus $9.7 \%$. The $31-45$ age group had a higher proportion of past-year drinkers; the proportion was the lowest in the 15-19 age group. Secondary education or a diploma was the education level with the highest proportion of past-year drinkers. Participants that had no exposure to alcohol advertisements represented a lower proportion of past-year drinkers than those who had exposure. A similar pattern was observed for past-year heavy drinking. Participants with male sex, working age, and secondary education or a diploma had a higher proportion of past-year heavy drinking compared to the other groups. A higher proportion of past-year heavy drinkers was observed in participants with exposure to alcohol advertisements than those without exposure, with the highest proportion represented by those who were uncertain about their exposure (Table 2).

Table 2. Proportions of past-year drinkers, past-year heavy drinkers, and past-year heavy drinkers among drinkers by characteristics.

\begin{tabular}{|c|c|c|c|}
\hline \multirow[b]{2}{*}{ Characteristic } & \multicolumn{3}{|c|}{$\%$} \\
\hline & $\begin{array}{c}\text { Past-Year } \\
\text { Drinkers } \\
(n=89,154)\end{array}$ & $\begin{array}{c}\text { Past-Year } \\
\text { Heavy } \\
\text { Drinkers } \\
(n=89,154)\end{array}$ & $\begin{array}{c}\text { Past-Year Heavy } \\
\text { Drinkers among } \\
\text { Drinkers } \\
(n=23,073)\end{array}$ \\
\hline Total & 25.9 & 10.4 & 40.0 \\
\hline \multicolumn{4}{|l|}{ Sex } \\
\hline Male & 45.1 & 20.2 & 44.7 \\
\hline Female & 9.7 & 2.1 & 21.5 \\
\hline \multicolumn{4}{|l|}{ Age (years) } \\
\hline $15-19$ & 12.1 & 4.8 & 39.5 \\
\hline $20-30$ & 31.5 & 14.1 & 44.8 \\
\hline $31-45$ & 34.4 & 14.8 & 42.9 \\
\hline $46-60$ & 29.2 & 11.3 & 38.9 \\
\hline $61+$ & 13.5 & 4.0 & 29.4 \\
\hline \multicolumn{4}{|l|}{ Education level } \\
\hline Primary education or lower & 23.5 & 9.0 & 38.5 \\
\hline Secondary education or diploma & 30.7 & 13.2 & 43.0 \\
\hline Bachelor's degree or higher & 23.7 & 8.6 & 36.1 \\
\hline Missing & 16.9 & 8.0 & 47.4 \\
\hline \multicolumn{4}{|l|}{$\begin{array}{l}\text { Self-reported exposure to } \\
\text { alcohol advertisements }\end{array}$} \\
\hline Exposed & 27.7 & 12.7 & 45.9 \\
\hline Not exposed & 24.8 & 8.9 & 35.9 \\
\hline Uncertain & 28.5 & 14.3 & 50.3 \\
\hline
\end{tabular}

\subsection{Factors Associated with Past-Year Drinking}

Table 3 displays the results of the regression model for past-year drinking (Model 1). Self-reported exposure to alcohol advertisements increased the odds of drinking by $16 \%$. 
Men were eight times more likely than women to be past-year drinkers. Participants in the working age groups were three to four times more likely to drink in the past year than those in the 61+ age group. Participants with the highest education level had slightly lower odds of drinking in the past year than those with primary education or lower, while those with secondary education or a diploma had higher odds of drinking.

Table 3. Factors associated with past-year drinking (Model 1; $n=89,154$ ).

\begin{tabular}{lccc}
\hline \multicolumn{1}{c}{ Factor } & OR & $\mathbf{9 5 \% ~ C I ~}$ & $p$-Value \\
\hline Self-reported exposure to alcohol advertisements & & & \\
$\quad$ Exposed & 1.16 & $1.07-1.27$ & 0.001 \\
Uncertain & $\begin{array}{r}1.02 \\
\text { ref }\end{array}$ & $0.99-1.06$ & 0.197 \\
Not exposed & & & \\
Sex & 8.33 & $8.02-8.65$ & 0.000 \\
Male & ref & & \\
Female & & & \\
Age (years) & 0.87 & $0.79-0.97$ & 0.013 \\
15-19 & 3.46 & $3.22-3.71$ & 0.000 \\
20-30 & 4.01 & $3.79-4.24$ & 0.000 \\
31-45 & 3.03 & $2.88-3.19$ & \\
46-60 & ref & & 0.000 \\
61+ & & & \\
Education level & 1.10 & $1.06-1.15$ & 0.000 \\
Secondary education or diploma & 0.82 & $0.76-0.87$ & 0.000 \\
Bachelor's degree or higher & 0.41 & $0.28-0.60$ & \\
Missing & ref & & \\
Primary education or lower & & & \\
\hline
\end{tabular}

\subsection{Factors Associated with Past-Year Heavy Drinking}

Table 4 presents the results of two regression models regarding the association between self-reported exposure to alcohol advertisements and past-year heavy drinking among the total participants (Model 2) and among past-year drinkers (Model 3). In Model 2, self-reported exposure to alcohol advertisements was associated with a $35 \%$ increase in the odds of past-year heavy drinking, whereas participants who were uncertain about their exposure had a $68 \%$ increase in the odds when compared to those who were not exposed. Men were 12 times more likely than women to be past-year heavy drinkers. The 31-45 age group had the highest odds of past-year heavy drinking. When compared to those with primary education or lower, those with the highest education level had lower odds of past-year heavy drinking, while those with secondary education or a diploma had higher odds of past-year heavy drinking.

In Model 3, self-reported exposure to alcohol advertisements was associated with a $51 \%$ increase in the odds of past-year heavy drinking. Past-year drinkers in all other age groups had higher odds of past-year heavy drinking than those aged 61+. Drinkers with a bachelor's degree or higher had lower odds of past-year heavy drinking than those in the lowest education group. 
Table 4. Factors associated with past-year heavy drinking.

\begin{tabular}{|c|c|c|c|c|c|c|}
\hline \multirow{2}{*}{ Factor } & \multicolumn{3}{|c|}{ Model $2(n=89,154)$} & \multicolumn{3}{|c|}{ Model $3(n=23,073)$} \\
\hline & OR & $95 \% \mathrm{CI}$ & $p$-Value & OR & $95 \%$ CI & $p$-Value \\
\hline \multicolumn{7}{|l|}{$\begin{array}{l}\text { Self-reported exposure to alcohol } \\
\text { advertisements }\end{array}$} \\
\hline Exposed & 1.35 & $1.28-1.41$ & 0.000 & 1.51 & $1.43-1.60$ & 0.000 \\
\hline Uncertain & 1.68 & $1.51-1.87$ & 0.000 & 1.74 & $1.52-1.99$ & 0.000 \\
\hline Not exposed & ref & & & ref & & \\
\hline \multicolumn{7}{|l|}{ Sex $11-5$} \\
\hline Male & 12.23 & $11.43-13.09$ & 0.000 & 3.12 & $2.89-3.37$ & 0.000 \\
\hline Female & ref & & & ref & & \\
\hline \multicolumn{7}{|l|}{ Age (years) } \\
\hline $15-19$ & 1.06 & $0.90-1.24$ & 0.488 & 1.34 & $1.11-1.62$ & 0.003 \\
\hline $20-30$ & 4.04 & $3.65-4.48$ & 0.000 & 1.91 & $1.69-2.15$ & 0.000 \\
\hline $31-45$ & 4.46 & $4.09-4.86$ & 0.000 & 1.93 & $1.74-2.13$ & 0.000 \\
\hline $46-60$ & 3.31 & $3.05-3.59$ & 0.000 & 1.65 & $1.51-1.82$ & 0.000 \\
\hline $61+$ & ref & & & ref & & \\
\hline \multicolumn{7}{|l|}{ Education level } \\
\hline Secondary education or diploma & 1.06 & $1.00-1.12$ & 0.042 & 0.96 & $0.90-1.02$ & 0.178 \\
\hline Bachelor's degree or higher & 0.73 & $0.68-0.80$ & 0.000 & 0.77 & $0.70-0.85$ & 0.000 \\
\hline Missing & 0.58 & $0.35-0.96$ & 0.033 & 1.11 & $0.58-2.13$ & 0.749 \\
\hline Primary education or lower & ref & & & ref & & \\
\hline
\end{tabular}

\section{Discussion}

In this study, the association between alcohol advertising exposure and drinking behaviors was investigated using data from a population-based survey. We demonstrate that self-reported exposure to alcohol advertisements was associated with past-year drinking and past-year heavy drinking adjusted for sex, age, and education. Male sex, working age, and secondary education or a diploma were associated with greater odds of past-year drinking and past-year heavy drinking.

The current study found that self-reported exposure to alcohol advertisements was associated with past-year drinking (OR, 1.16; 95\% CI, 1.07-1.27), past-year heavy drinking (OR, 1.35; 95\% CI, 1.28-1.41), and past-year heavy drinking among drinkers (OR, 1.51; 95\% CI, 1.43-1.60) in participants aged 15 or older. Similarly, a previous cross-sectional survey of 1200 university students in Bangkok, Thailand, reported a positive association between self-reported alcohol advertising exposure and the frequency of past-year drinking [14]. Self-reported exposure to alcohol advertising increased the likelihood of ever drinking (relative risk (RR), 1.41; 95\% CI, 1.10-1.80) and being problematic drinkers (RR, $1.85 ; 95 \%$ CI $1.40-2.44)$ in a study of 1076 Chilean adolescents [2]. An analysis of a cross-sectional survey of 3806 school children in Cambodia found that almost daily/daily exposure to alcohol advertising (self-reported) was associated with alcohol use in the last 30 days (OR, 1.61; 95\% CI, 1.03-2.51) and ever having drunkenness (OR, 2.30; 95\% CI, 1.40-3.77) [3]. An online cross-sectional survey of 3999 UK adolescents reported that a medium to high level of self-reported alcohol marketing exposure (referred to as "alcohol marketing awareness" in the original article) was associated with high-risk drinking (i.e., AUDIT-C score $\geq 5$; OR, 1.43-2.18) among past-year drinkers, but not with susceptibility to drink among teetotalers [6]. Summarily, self-reported exposure to alcohol advertisements was positively associated with various measures of drinking behaviors, with the exception of teetotalers' susceptibility to drink.

Whereas the studies discussed in the preceding paragraph used self-reported exposure to any types of alcohol advertising as the exposure variable in multivariate analysis, there were studies that examined the effects of exposure to specific types of alcohol advertising or marketing. A cross-sectional study of 955 participants from a municipality in South Africa reported that SMS advertising and free alcohol offers were associated with a higher chance of heavy drinking in the past six months. Large posters/billboards, sport and 
music sponsorship, signs or posters, TV, radio, famous people, magazines/newspapers, and email advertising, on the other hand, were not associated with heavy drinking [4]. A cross-sectional study of 4413 adolescents in Victoria, Australia, found that self-reported exposure (awareness) to alcohol advertising via billboards/newspapers/magazines and alcohol merchandise ownership increased the chance of past-month alcohol use and pastweek heavy drinking. Past-month alcohol use and past-week heavy drinking were not associated with advertising on television, at sporting events, on the internet, or through sports sponsorship [15]. An online cross-sectional survey of 9038 school students from four European countries reported that self-reported online alcohol advertising exposure and ownership of alcohol-branded items were associated with an increased chance of ever drinking and past-month heavy drinking. This study also found a dose-response relationship between overall exposure to alcohol advertisements and ever drinking and past-month heavy drinking [5]. A cross-sectional study of 2257 school students in Zambia found that being given free alcohol was associated with ever having drunkenness and ever having drinking-related problems. The study found no association between other types of alcohol marketing (i.e., using actors and billboards) and either drinking outcome [16]. These findings suggest that different types of advertising or marketing activities may have varying effects on drinking behaviors. This could be due to the differences in the context of each study. It could also be because of a qualitative aspect of the advertisement, such as its design, provision, and timing. Nonetheless, free alcohol offers and ownership of alcohol merchandise were consistently associated with drinking behaviors across studies (where these types of advertising were included as exposure variables).

The proportion of participants in this study who were exposed to alcohol advertisements was $32.7 \%$, which was lower than the proportions reported in other studies. The percentage of past-month exposure to alcohol advertising in Cambodian school children was $81.4 \%$ [3]. More than $40 \%$ of UK adolescents were exposed to alcohol advertising on television in the past week [6]. Almost half of the participants in a survey of South Africans reported that they were frequently exposed to alcohol advertisements [4]. This was likely due to the effect of Section 32 of Thailand's Alcoholic Beverage Control Act, B.E. 2551 (2008), which prohibits most forms of alcohol advertising.

Our findings are in agreement with those of other studies, which found that exposure to alcohol advertisements was generally associated with a higher chance of drinking and heavy drinking. Due to a lack of information on each type of alcohol advertising exposure in the SADBeS17, we were unable to explore the effect of each type of advertising, which has been shown to have a different effect on drinking behaviors. Most studies on this topic were conducted using data from adolescents or young adults, whereas the data used in this study were collected from the general population aged 15 years or older. As a result, the findings of this study can be generalized to a wider population compared to those from previous studies.

Our finding that men were more likely to be past-year drinkers and past-year heavy drinkers fits with the general pattern of drinking behaviors [1]. The findings on drinking and heavy drinking by age group are also consistent with drinking patterns in middleincome Asian countries, where adolescents and the elderly had less likelihood of being past-year drinkers and heavy drinkers than working-age populations [1,17]. In general, socioeconomic status is associated with greater alcohol consumption [1]. The relationship between education level (one of the indicators of socioeconomic status) and past-year drinking and past-year heavy drinking did not follow that pattern. Participants in the current study who had secondary education or a diploma had the highest odds of being past-year drinkers and past-year heavy drinkers. Nonetheless, this pattern was observed in a study that analyzed data from two population-based surveys conducted in Serbia and Hungary. Survey participants with secondary education had the highest chance of past-year drinking. This pattern was observed in both countries [18]. A study conducted in the United States reported a positive association between education and ever drinking. The sub-group analysis revealed that this pattern was found in Whites, but not in African 
American participants. African American participants with a high school diploma had a higher chance of ever drinking than those with no diploma or a college education [19]. This demonstrates that the relationship between education level and drinking varies across countries or populations.

The major strength of this study is that it used data from a population-based survey, whereas most studies exploring the relationship between alcohol advertisement and drinking behaviors used data from a narrow age range (adolescents and/or young adults). The findings of this study can be applied to a wider population. This study does have some limitations. The exposure to alcohol advertisements was determined by self-report. As a result, some participants may be unaware of their exposure, but it may still affect their drinking behaviors. This issue was partially resolved by having "uncertain" as a level of the exposure variable. Participants who were unsure about their exposure were more similar to those with exposure to alcohol advertisements than those without exposure in terms of their drinking behaviors. The survey used a 12-month duration for assessment of drinking behavior. This duration is relatively long, and it is possible that participants may change their drinking pattern during this period. The past 30-day or 4-week duration, as employed in several other studies, should be considered in future research on this topic. The survey did not collect information on exposure to each type of advertisement. As shown in a number of studies that different types of advertising and marketing had a varying magnitude of effect on alcohol consumption, data on the types and degree of advertising exposure should be collected and used as explanatory variables to enhance the understanding of the effect of alcohol advertising and marketing on alcohol consumption in Thailand. Another limitation was the cross-sectional nature of the data, which did not allow causal inference. Only the association between variables could be examined. Further studies on this topic in Thailand should employ a longitudinal design and include exposure to different types of advertisements.

\section{Conclusions}

Using data from a population-based survey in Thailand, a middle-income country with strict regulations on alcohol advertisements, this analysis shows that self-reported exposure to alcohol advertisements was associated with increasing odds of past-year drinking and past-year heavy drinking. Further studies exploring the effect of each type of advertisement using more sensitive alcohol consumption measurements are encouraged to gain more insight into the effect of alcohol advertisements on alcohol consumption.

Author Contributions: Conceptualization, P.B. and U.S.; methodology, P.B. and U.S.; software, P.B. and U.S.; validation, U.S.; formal analysis, P.B.; data curation, P.B. and U.S.; writing-original draft preparation, P.B.; writing - review and editing, U.S.; supervision, U.S.; funding acquisition, U.S. All authors have read and agreed to the published version of the manuscript.

Funding: This research was funded by the Center for Alcohol Studies, Thailand, grant number 62-02029-0043.

Institutional Review Board Statement: This study was conducted according to the guidelines of the Declaration of Helsinki and approved by the Human Research Ethics Committee of Walailak University (WU-EC-MD-3-473-63).

Informed Consent Statement: Informed consent was obtained from all survey participants. For participants aged less than 18 years, consent was given by their guardians and themselves.

Data Availability Statement: The authors obtained the data used in this study from the Center for Alcohol Studies with the permission under the contract of this study. Request for the data can be made to the corresponding author (saengow.udomsak@gmail.com). Access to the data must be permitted by the Center for Alcohol Studies.

Conflicts of Interest: The authors declare no conflict of interest. 


\section{References}

1. World Health Organization. Global Status Report on Alcohol and Health 2018; World Health Organization: Geneva, Switzerland, 2019.

2. Sanhueza, G.E.; Delva, J.; Bares, C.B.; Grogan-Kaylor, A. Alcohol consumption among Chilean adolescents: Examining individual, peer, parenting and environmental factors. Int. J. Alcohol Drug Res. 2013, 2, 89. [CrossRef] [PubMed]

3. Peltzer, K.; Pengpid, S.; Tepirou, C. Associations of alcohol use with mental health and alcohol exposure among school-going students in Cambodia. Nagoya J. Med. Sci. 2016, 78, 415.

4. Williams, P.P.; Morojele, N.; Londani, M.; Harker Burnhams, N.; Parry, C.D. Alcohol advertising, affordability and availability, and the effect on adult heavy drinking and symptoms of alcohol problems: International alcohol control study (South Africa). Subst. Use Misuse 2019, 54, 1751-1762. [CrossRef] [PubMed]

5. de Bruijn, A.; Engels, R.; Anderson, P.; Bujalski, M.; Gosselt, J.; Schreckenberg, D.; Wohtge, J.; de Leeuw, R. Exposure to online alcohol marketing and adolescents' drinking: A cross-sectional study in four European countries. Alcohol Alcohol. 2016, 51, 615-621. [CrossRef] [PubMed]

6. Critchlow, N.; MacKintosh, A.M.; Thomas, C.; Hooper, L.; Vohra, J. Awareness of alcohol marketing, ownership of alcohol branded merchandise, and the association with alcohol consumption, higher-risk drinking, and drinking susceptibility in adolescents and young adults: A cross-sectional survey in the UK. BMJ Open 2019, 9, e025297. [CrossRef] [PubMed]

7. Niederdeppe, J.; Avery, R.J.; Tabor, E.; Lee, N.W.; Welch, B.; Skurka, C. Estimated televised alcohol advertising exposure in the past year and associations with past 30-day drinking behavior among American adults: Results from a secondary analysis of large-scale advertising and survey data. Addiction 2021, 116, 280-289. [CrossRef] [PubMed]

8. Jernigan, D.; Noel, J.; Landon, J.; Thornton, N.; Lobstein, T. Alcohol marketing and youth alcohol consumption: A systematic review of longitudinal studies published since 2008. Addiction 2017, 112, 7-20. [CrossRef] [PubMed]

9. Noel, J.K.; Sammartino, C.J.; Rosenthal, S.R. Exposure to digital alcohol marketing and alcohol use: A systematic review. J. Stud. Alcohol Drugs 2020, S 19, 57-67. [CrossRef]

10. Saengow, U. Thailand's national alcohol survey as a policy monitoring tool. Drugs Alcohol Today 2017, 17, 250-257. [CrossRef]

11. Government Gazette. Alcoholic Beverage Control Act, B.E. 2551. 2008. Available online: http://web.krisdika.go.th/data/ outsitedata/outsite21/file/ALCOHOLIC_BEVERAGE_CONTROL_ACT,_B.E._2551_(2008).pdf (accessed on 30 August 2021).

12. Limstit, P.; Chalidabhongse, J.; Dangsakul, J. Study on Legal Issues of Brand DNA under Trade Mark Law and Recommendations to Amend the Alcohol Beverage Controla Act, B.E. 2551. Available online: http://cas.or.th/cas/wp-content/uploads/2021/01/ $\%$ E0\%B8\%A3\%E0\%B8\%B2\%E0\%B8\%A2\%E0\%B8\%87\%E0\%B8\%B2\%E0\%B8\%99-\%E0\%B8\%81\%E0\%B8\%8E\%E0\%B8\%AB \%E0 \%B8\%A1\%E0\%B8\%B2\%E0\%B8\%A2-Brand-DNA-\%E0\%B9\%84\%E0\%B8\%9E\%E0\%B8\%A8\%E0\%B8\%B2\%E0\%B8\%A5-final.pdf (accessed on 25 August 2021).

13. Saengow, U. Drinking abstinence during a 3-month abstinence campaign in Thailand: Weighted analysis of a national representative survey. BMC Public Health 2019, 19, 1-8. [CrossRef] [PubMed]

14. Vantamay, S. Alcohol consumption among university students: Applying a social ecological approach for multi-level preventions. Southeast Asian J. Trop. Med. Public Health 2009, 40, 354.

15. Faulkner, A.; Azar, D.; White, V. ‘Unintended'audiences of alcohol advertising: Exposure and drinking behaviors among Australian adolescents. J. Subst. Use 2017, 22, 108-112. [CrossRef]

16. Swahn, M.H.; Ali, B.; Palmier, J.B.; Sikazwe, G.; Mayeya, J. Alcohol marketing, drunkenness, and problem drinking among Zambian youth: Findings from the 2004 Global School-Based Student Health Survey. J. Environ. Public Health 2011, $2011,497827$. [CrossRef] [PubMed]

17. Chaiyasong, S.; Huckle, T.; Mackintosh, A.M.; Meier, P.; Parry, C.D.H.; Callinan, S.; Viet Cuong, P.; Kazantseva, E.; Gray-Phillip, G.; Parker, K.; et al. Drinking patterns vary by gender, age and country-level income: Cross-country analysis of the International Alcohol Control Study. Drug Alcohol Rev. 2018, 37, S53-S62. [CrossRef] [PubMed]

18. Mihailovic, N.; Szőlloósi, G.J.; Rancic, N.; János, S.; Boruzs, K.; Nagy, A.C.; Timofeyev, Y.; Dragojevic-Simic, V.; Antunovic, M.; Reshetnikov, V. Alcohol consumption among the elderly citizens in Hungary and Serbia-Comparative assessment. Int. J. Environ. Res. Public Health 2020, 17, 1289. [CrossRef] [PubMed]

19. Assari, S.; Lankarani, M.M. Education and alcohol consumption among older Americans; black-white differences. Front. Public Health 2016, 4, 67. [CrossRef] [PubMed] 